\title{
Improving haemodynamic optimization of cardiac resynchronization therapy for heart failure
}

\begin{abstract}
Alexander J Sharp ${ }^{\mathrm{a}}$ MBBS MRCP, S M Afzal Sohaib ${ }^{\mathrm{b}, \mathrm{e}}$ MBBS MRCP, Matthew J Shun-Shin ${ }^{\mathrm{b}}$ MA MB BChir MRCP, Punam Pabari ${ }^{\mathrm{b}}$ MBChB MRCP PhD, Keith Willson ${ }^{\mathrm{b}}$ MSc, Christopher Rajkumar ${ }^{\mathrm{b}}$, Alun D Hughes ${ }^{\mathrm{c}}$ MBBS PhD, Prapa Kanagaratnam ${ }^{\mathrm{b}}$ FRCP PhD, Jamil Mayet ${ }^{\mathrm{b}}$ MBCHB MD MBA FESC FACC FRCP, Zachary I Whinnett $^{\mathrm{b}}$ PhD MRCP, Andreas A Kyriacou ${ }^{\mathrm{d}}$ MBChB PhD MRCP, Darrel P Francis ${ }^{\mathrm{b}}$ MA FRCP
\end{abstract}

\begin{abstract}
a. Addenbrooke's Hospital, Cambridge University Hospitals NHS Trust, Hills Rd, Cambridge CB2 0QQ, UK
b. National Heart \& Lung Institute, Imperial College London, Hammersmith Hospital Campus, Ducane Road, London, W12 0HS, UK

c. Institute of Cardiovascular Science, University College London, Gower Street, London, WC1E 6BT, UK

d. Department of Cardiology, Northern General Hospital, Sheffield Teaching Hospitals NHS Trust, Herries Rd, Sheffield S5 7AU, UK

e. Department of Cardiac Electrophysiology, Bart's Heart Centre, St Bartholomew's Hospital, West Smithfield, London EC1A 7BE
\end{abstract}

All work was performed at National Heart \& Lung Institute, Imperial College London.

Correspondence to:

Dr S M Afzal Sohaib

International Centre for Circulatory Health, 2nd Flr, B Block South, Hammersmith Campus, Imperial College

London, Ducane Road, London, W12 0HS, UK

Email: s.sohaib@imperial.ac.uk

Tel: +44 (0) 7904109057

Manuscript word count: 2,633 


\begin{abstract}
Objective

Optimization of cardiac resynchronization therapy using non-invasive haemodynamic parameters, produces reliable optima when performed at high atrial paced heart rates. Here we investigate whether this is a result of increased heart rate or atrial pacing itself.
\end{abstract}

\title{
Approach
}

43 patients with cardiac resynchronization therapy underwent haemodynamic optimization of AV delay using non-invasive beat-to-beat systolic blood pressure in three states: rest (atrial-sensing, $66 \pm 11 \mathrm{bpm}$ ), slow atrial pacing $(73 \pm 12 \mathrm{bpm})$, and fast atrial pacing $(94 \pm 10 \mathrm{bpm})$. A 20 -patient subset underwent a fourth optimization, during exercise $(80 \pm 11 \mathrm{bpm})$.

\section{Main results}

Intraclass correlation coefficient (ICC, quantifying information content mean \pm SE) was $0.20 \pm 0.02$ for resting sensed optimization, $0.45 \pm 0.03$ for slow atrial pacing ( $\mathrm{p}<0.0001$ versus rest-sensed), and $0.52 \pm 0.03$ for fast atrial pacing ( $\mathrm{p}=0.12$ versus slow-paced). $78 \%$ of the increase in ICC, from sinus rhythm to fast atrial pacing, is achieved by simply atrially pacing just above sinus rate.

Atrial pacing increased signal (blood pressure difference between best and worst AV delay) from $6.5 \pm 0.6 \mathrm{mmHg}$ at rest to $13.3 \pm 1.1 \mathrm{mmHg}$ during slow atrial pacing $(\mathrm{p}<0.0001)$ and $17.2 \pm 1.3 \mathrm{mmHg}$ during fast atrial pacing ( $\mathrm{p}=0.003$ versus slow atrial pacing).

Atrial pacing reduced noise (average SD of systolic blood pressure measurements) from $4.9 \pm 0.4 \mathrm{mmHg}$ at rest to $4.1 \pm 0.3 \mathrm{mmHg}$ during slow atrial pacing $(\mathrm{p}=0.28)$. At faster atrial pacing the noise was $4.6 \pm 0.3 \mathrm{mmHg}(\mathrm{p}=0.69$ versus slow-paced, $\mathrm{p}=0.90$ versus rest-sensed).

In the exercise subgroup ICC was $0.14 \pm 0.02$ ( $\mathrm{p}=0.97$ versus rest-sensed). 


\title{
Significance
}

\begin{abstract}
Atrial pacing, rather than the increase in heart rate, contributes to $\sim 80 \%$ of the observed information content improvement from sinus rhythm to fast atrial pacing. This is predominantly through increase in measured signal.
\end{abstract}

\section{Key Words}

Heart failure; cardiac resynchronization therapy; haemodynamic optimization; atrioventricular delay 


\section{Introduction}

Current guidelines recommend atrioventricular (AV) optimization of cardiac resynchronization therapy (CRT) devices be conducted at resting heart rates, and do not specify whether it is worthwhile to also perform optimization during atrial pacing ${ }^{1,2}$. Optimization using blood pressure (BP) with continuous finger photoplethysmography (Finometer), is a rapid and less labour intensive method than echocardiography based protocols and we have demonstrated it is non-inferior to this method in a randomised controlled trial ${ }^{3}$. Studies using this technique have shown optimization is more reliable when it is performed with faster atrial pacing ${ }^{4}$. Faster, atrially paced heart rates produce a greater signal ${ }^{4}$ and signal-to-noise ratio ${ }^{5}$ during photoplethysmographic optimization, and an analysis of previously published studies ${ }^{6}$ demonstrated a positive correlation between intraclass correlation coefficient (ICC) and increased heart rate during the optimization process regardless of protocol used.

This study investigates whether any improvement observed in the reliability of optimization with faster, atrially paced heart rates, is due solely to the increase in heart rate, or whether the change in atrial activation from native to paced is responsible. We investigated this by assessing signal to noise characteristics of optimizations performed at resting sinus rate, during a slowly paced atrial rate, and at higher heart rates by faster atrial pacing and, in a subset of patients, during exercise. 


\section{Methods}

\section{Participants}

43 consecutive outpatients with biventricular pacemakers or biventricular defibrillators implanted for clinical indications were enrolled in this study (Table 1). The only exclusion criteria were presence of atrial fibrillation, or an inability for the patient to comfortably lie flat for an extended period. Patients gave written informed consent for this study, which complied with the Declaration of Helsinki and was approved by the local ethics committee.

\begin{tabular}{|c|c|}
\hline \multicolumn{2}{|c|}{ Table 1. Patient characteristics } \\
\hline Characteristic & $\begin{array}{c}\text { Mean } \pm \text { SD or } \\
\text { Number (\%) }\end{array}$ \\
\hline Age & $67 \pm 10$ \\
\hline Male & $26(60)$ \\
\hline \multicolumn{2}{|l|}{ NYHA Class: } \\
\hline II & $19(44)$ \\
\hline III & $20(47)$ \\
\hline IV & $3(7)$ \\
\hline \multicolumn{2}{|l|}{ Aetiology of Heart Failure: } \\
\hline Ischemic cardiomyopathy & $17(40)$ \\
\hline Non-ischaemic Cardiomyopathy & $26(60)$ \\
\hline Left Ventricular Ejection Fraction & $28 \pm 8$ \\
\hline \multicolumn{2}{|l|}{ Drugs: } \\
\hline$\beta$-blocker & $39(91)$ \\
\hline $\begin{array}{r}\text { ACE inhibitor/Angiotensin receptor } \\
\text { blocker }\end{array}$ & $40(93)$ \\
\hline Diuretic (Loop diuretic/spironolactone) & $32(74)$ \\
\hline Digoxin & $4(9)$ \\
\hline
\end{tabular}




\section{Data collection}

Non-invasive, beat-to-beat BP was measured using a Finometer (Finapres Medical Systems, Amsterdam,

Netherlands). This system utilises an inflating finger cuff and volume-clamp photoplethysmography to produce a continuous arterial pressure waveform. Pressure exerted by the cuff is automatically adjusted, such that a constant blood volume within the finger (as measured by photoplethysmography) is maintained. Thus, pressure exerted by the cuff is a surrogate of intra-arterial pressure ${ }^{4,5,7,8,9,10}$.

An ECG signal was recorded using a Hewlett-Packard 78351A monitor. Analogue signals were taken via a National Instruments DAQ-Card AI-16E-4 (National Instruments, Austin, TX, USA) and Labview (National Instruments, Austin, TX, USA).

Off line analysis was performed using custom software based on the Matlab platform (MathWorks, Natick, MA, $\mathrm{USA})^{11}$.

\section{AV optimization protocol}

The optimization protocol involves defining at which AV delay systolic BP (SBP) is greatest. SBP is plotted against the AV delay, and a parabolic curve is fitted. The AV delay identified as the peak of the parabola is selected as the AV optimum. To minimise the effects of any spontaneous fluctuations in BP, absolute SBP values were not measured; instead, change in SBP between a fixed reference AV delay (such as AV 120ms) and a number of pre-specified AV delays were tested ${ }^{4,5,7,8}$. Tested AV delays were 40, 80, 140, 160, 200, 240,280 and 300ms, but only included those delays which produced ventricular capture, i.e. excluded the long AV delays which allowed solely intrinsic ventricular activation.

At each tested AV delay, the change in SBP from the reference AV delay was determined by taking the mean SBP of 10 beats immediately post-transition, and subtracting the mean SBP of 10 beats immediately pretransition. By taking an average of 10 beats, effect of respiratory noise and other periodic fluctuations in BP were reduced s,5,7,8. $^{4}$

Transitions between each tested AV delay and the reference were repeated, with an equal number of forward (reference to tested delay) and backwards (tested delay to reference) transitions, and absolute values of mean 
change in SBP were calculated. In this way, any upwards or downwards trend in BP during the measurement were negated. At all tested AV delays (at all rates) we performed the same number of transitions (8 transitions) to enable valid comparisons of Intraclass Correlation Coefficient (ICC) $)^{4,5,7,8}$.

To calculate optimal AV delay from the data recorded, values for AV delay were plotted against mean SBP change of the 8 transitions. A quadratic curve was fitted, the peak of which was taken as representing optimal AV delay.

\section{Atrial Pacing versus heart rate}

To assess the impact of heart rate versus atrial pacing on the accuracy of AV optimum value obtained, the 43 patients had the optimization procedure repeated at three pacing modes. From these, signal, noise and intraclass correlation coefficient (ICC) were calculated:

1. Atrial sensed, resting heart rate (rest-sensed)

2. Atrial paced, at the lowest programmable rate above resting sinus rate that generated consistent atrial capture (slow-paced)

3. Atrial paced $\sim 25 \mathrm{bpm}$ above slow-paced (fast-paced)

In addition, 20 patients had the optimization procedure performed during exercise to give a non-paced elevated heart rate (exercise-sensed). To enable easy measurements using the Finometer, participants were exercised on a supine bicycle (Medical Positioning Inc, Kansas City, MO, USA). From this group, signal, noise and intraclass correlation coefficient (ICC) were also calculated.

\section{Signal and noise}

For all of the tested AV delays at each heart rate, the mean change in SBP for the 8 transitions and the standard error of the mean (SEM) were calculated. In this study, signal was defined as the difference in SBP between the worst and optimal AV delays, and noise by the average of all the standard deviations (SD) of the mean change of systolic blood pressure, at all tested AV delays in each pacing mode. 


\section{Intraclass correlation coefficient}

Intraclass correlation coefficient (ICC) provides a measure of information content similar to the signal-to-noise ratio. It has the major advantage of varying between 0 and 1 , as opposed to extending to infinity ${ }^{6}$. This makes it a more tangible concept, with zero indicating that a measurement is just noise, and at the other end of the spectrum, 1 indicating pure signal and no noise.

ICC is equal to the ratio of signal variance to the total variance of signal and noise ${ }^{6}$ (box 1). For practical purposes, in this study we have defined signal variance as the between-individual variance between the means at each individual AV setting. The total variance is the variance of all the data points from all individuals which contribute to these means and is the sum of the between and within individual variance. If all the measurements at any particular AV delay setting within an individual are identical, then these two variances are the same, i.e. ICC $=1$. At the other extreme, if all the measurements at each setting are show considerable variance within an individual and this accounts for almost all of the total variance, then the ICC will be almost zero.$$
\mathrm{ICC}=\frac{\mathrm{R}}{\mathrm{R}-1} \frac{\mathrm{Vm}}{\mathrm{Vraw}}-\frac{1}{\mathrm{R}-1}
$$

Box 1. Calculation of ICC

$\mathrm{R}=$ number of replicate sets of optimisations in patient

Vraw $=$ variance of all raw measurements

$\mathrm{Vm}=$ variance of mean measurement at each AV delay

\section{Assessment of curvature}

Optimization curves were constructed using least squares fitting to a parabola. The degree of curvature of the optimization response was quantified by the curvature coefficient of the fitted curve, i.e. the "a" from the quadratic formula $a x^{2}+b x+c$.

\section{Statistics}

Data are presented as mean \pm SEM. To quantify the differences between the different pacing configurations a two way ANOVA was performed with patient and pacing configuration as factors. Where significant we used post hoc testing with Tukey's HSD. A p-value $<0.05$ was considered statistically significant. Statistical analysis was performed using R (R Foundation for Statistical Computing, Vienna, Austria). 


\section{Results}

\section{Separating the impact of atrial pacing from the impact of increased heart rate}

The change from resting (atrial sensed) to fast atrial pacing provides two contributions: the regularization of heart rate by institution of atrial pacing, and the substantial increase in heart rate.

The atrial pacing contribution, defined as the rise in ICC from resting to slow paced, was large: from $0.20 \pm 0.02$ to $0.45 \pm 0.03$ ( $\mathrm{p}<0.0001)$. It should be remembered that this contains a small component of heart rate increase, from $66 \pm 11 \mathrm{bpm}$ (rest-sensed) to $73 \pm 12 \mathrm{bpm}$ (slow-paced).

The contribution from the substantial increase in heart rate, defined as the rise in ICC from slow atrial pacing ( $73 \pm 12 \mathrm{bpm})$ to fast atrial pacing $(94 \pm 10 \mathrm{bpm})$, was smaller: from $0.45 \pm 0.03$ to $0.52 \pm 0.03$ ( $\mathrm{p}=0.12)$.

On this basis, the contributions to the increase in optimization efficiency provided by fast atrial pacing (instead of rest) could be summarised as $78 \%$ from institution of atrial pacing plus $22 \%$ from the substantial increase in heart rate.

Attempting to raise the ICC by increasing heart rate substantially without atrial pacing (i.e. by exercise) was not successful: ICC showed no sign of rising, going from $0.20 \pm 0.02$ to $0.14 \pm 0.02$ ( $\mathrm{p}=0.9$, Figure 1 ). The average exercise-sensed heart rate achieved was $80 \pm 11 \mathrm{bpm}$. 


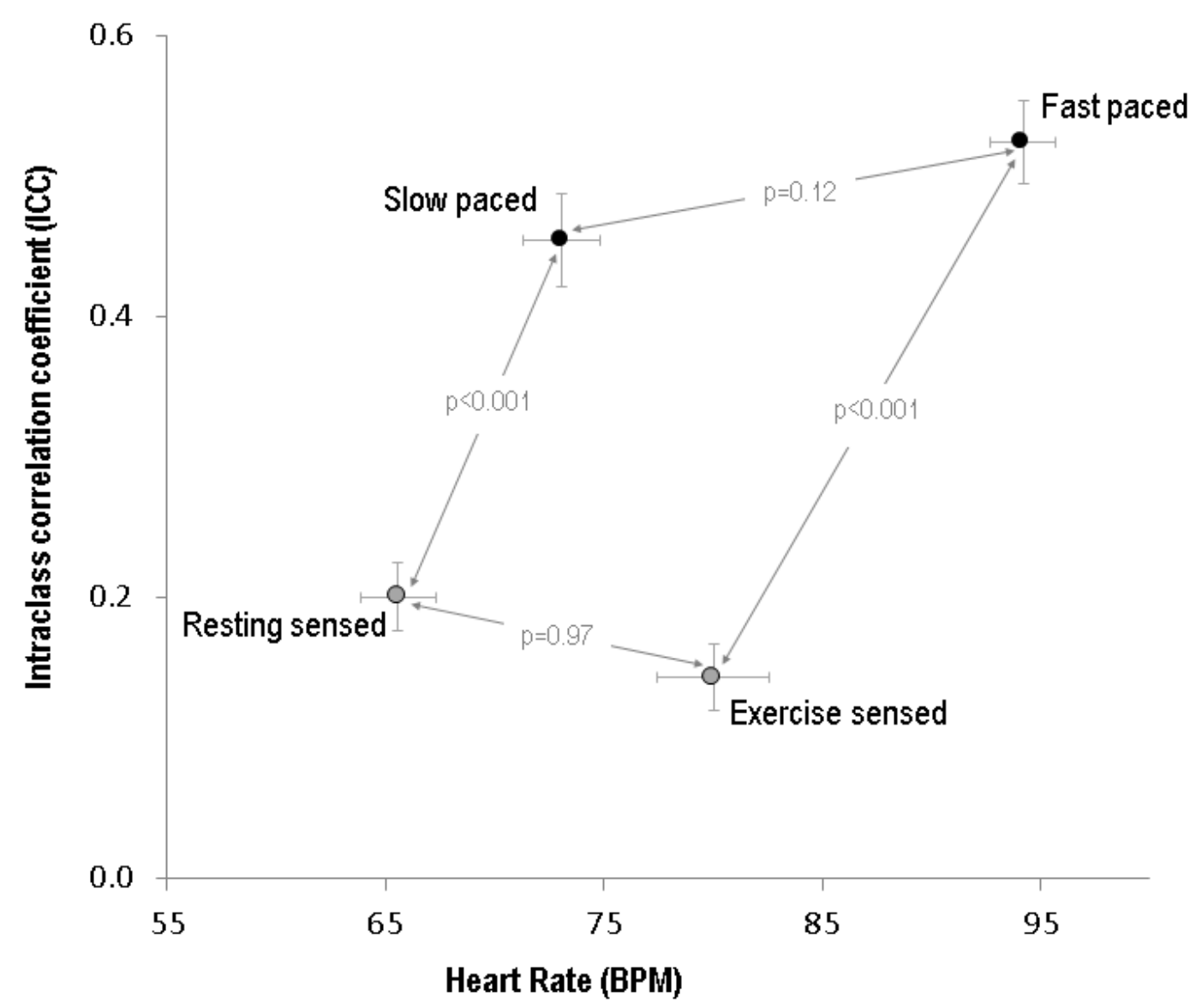

Figure 1. Effect of the atrial pacing mode and heart rate on the Intraclass Correlation Coefficient (ICC) Atrial pacing significantly improves information content, and is primarily responsible for the large increase in ICC seen between resting sensed and fast paced optimizations. The ICC did not improve during exercise.

\section{Does improvement in signal-to-noise ratio arise from increase in signal or decrease in noise?}

For optimization, the relevant signal is the size of the difference in SBP between different AV delays. A simple quantification of this is the difference between the highest and lowest.

The atrial pacing component increased measured signal from $6.5 \pm 0.6 \mathrm{mmHg}$ during rest-sensed to 13.3 $\pm 1.1 \mathrm{mmHg}$ during slow-paced $(\mathrm{p}<0.0001)$. At the higher rates the atrial pacing component increased signal from $4.7 \pm 0.6 \mathrm{mmHg}$ during exercise-sensed to $17.2 \pm 1.3 \mathrm{mmHg}$ during fast-paced $(\mathrm{p}<0.0001$ ), as shown in the upper panel of Figure 2. 
2

The heart rate increase component improved measured signal from $13.3 \pm 1.1 \mathrm{mmHg}$ during slow-paced to $17.2 \pm 1.3 \mathrm{mmHg}$ during fast-paced $(\mathrm{p}=0.003)$. Without atrial pacing, there was no evidence of an increase in signal when heart rate was increased: $6.5 \pm 0.6 \mathrm{mmHg}$ at rest, and $4.7 \pm 0.6 \mathrm{mmHg}$ on exercise $(\mathrm{p}=0.8)$ as shown in lower panel of Figure 2.

On this basis, the contributions to the increase in signal provided by fast atrial pacing (instead of rest) could be summarised as $78 \%$ from institution of atrial pacing plus $22 \%$ from the substantial increase in heart rate.

Meanwhile the relevant noise is the uncertainty in the measurement of the SBP at each AV delay, which can be quantified as the average of the SDs across all AV delays. There was no statistically significant decrease in noise with the initiation of atrial pacing $(\mathrm{p}=0.28)$ nor between rest and fast pacing (Figure 2 lower panel). 


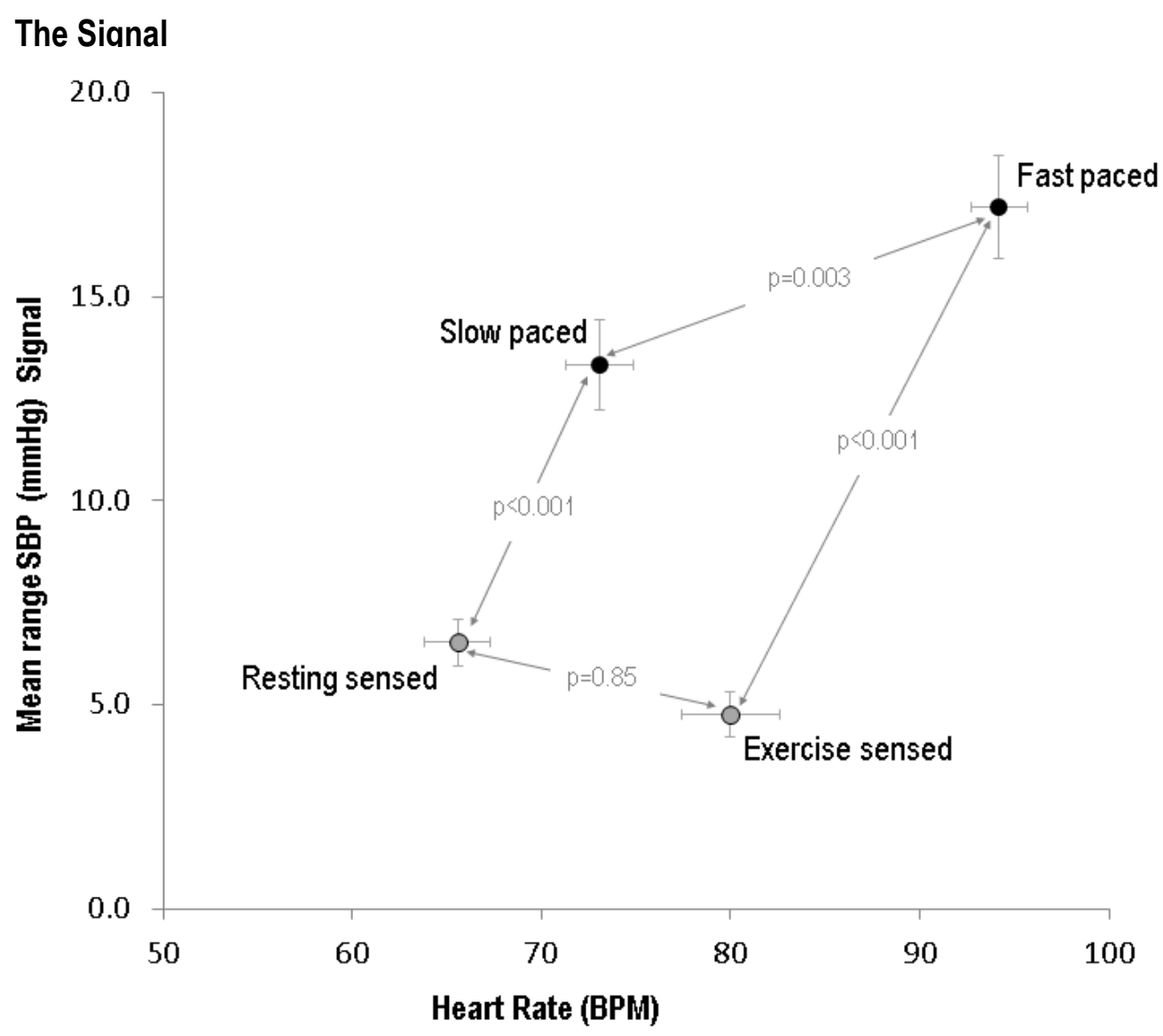

The Noise

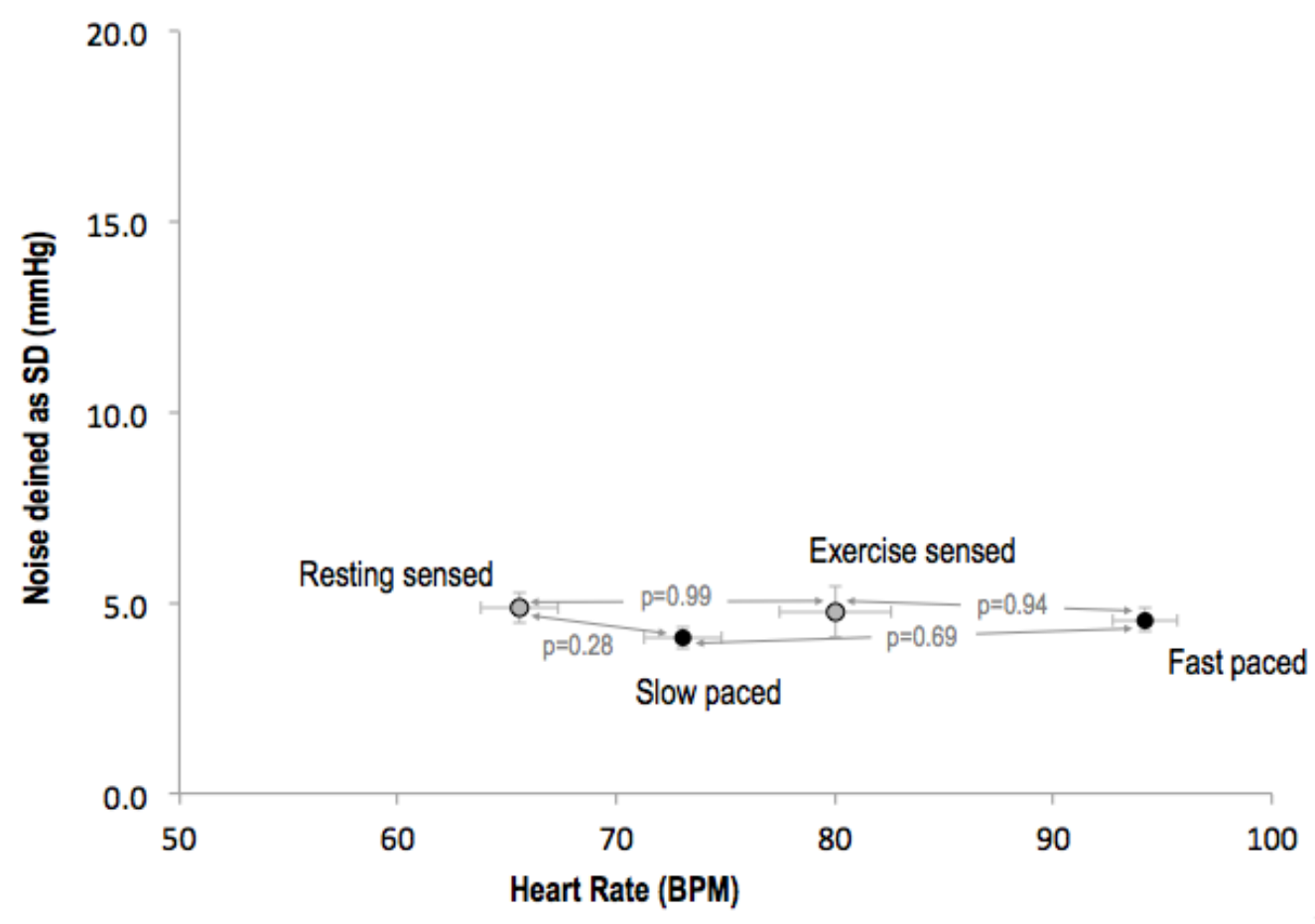

Figure 2. Contributions from the institution of atrial pacing mode and of increase in heart rate on signal and noise in haemodynamic optimization 


\section{Parabola Curvature}

Identifying an optimal AV delay with precision (i.e. with a small uncertainty) requires a strong curvature of the haemodynamic response when plotted against AV delay ${ }^{12,13}$.

There was no evidence that atrial pacing affected curvature; curvature was $3.8 \pm 0.6 \times 10^{-4} \mathrm{~mm} / \mathrm{Hg} / \mathrm{s}^{2}$ at rest and $4.9 \pm 0.5 \times 10^{-4} \mathrm{~mm} / \mathrm{Hg} / \mathrm{s}^{2}$ with slow pacing $(\mathrm{p}=0.38)$ as shown in Figure 3 .

The effect of an increase in heart rate, defined as the increase in curvature from slow atrial pacing to fast atrial pacing, was substantial: from $4.9 \pm 0.5 \times 10^{-4} \mathrm{~mm} / \mathrm{Hg} / \mathrm{s}^{2}$ to $7.2 \pm 0.5 \times 10^{-4} \mathrm{~mm} / \mathrm{Hg} / \mathrm{s}^{2} \quad(\mathrm{p}=0.006)$.

Increasing heart rate substantially without atrial pacing (i.e. by exercise) did not increase curvature ( $p=0.40$ vs rest and $\mathrm{p}=0.99$ vs slow atrial pacing, Figure 3).

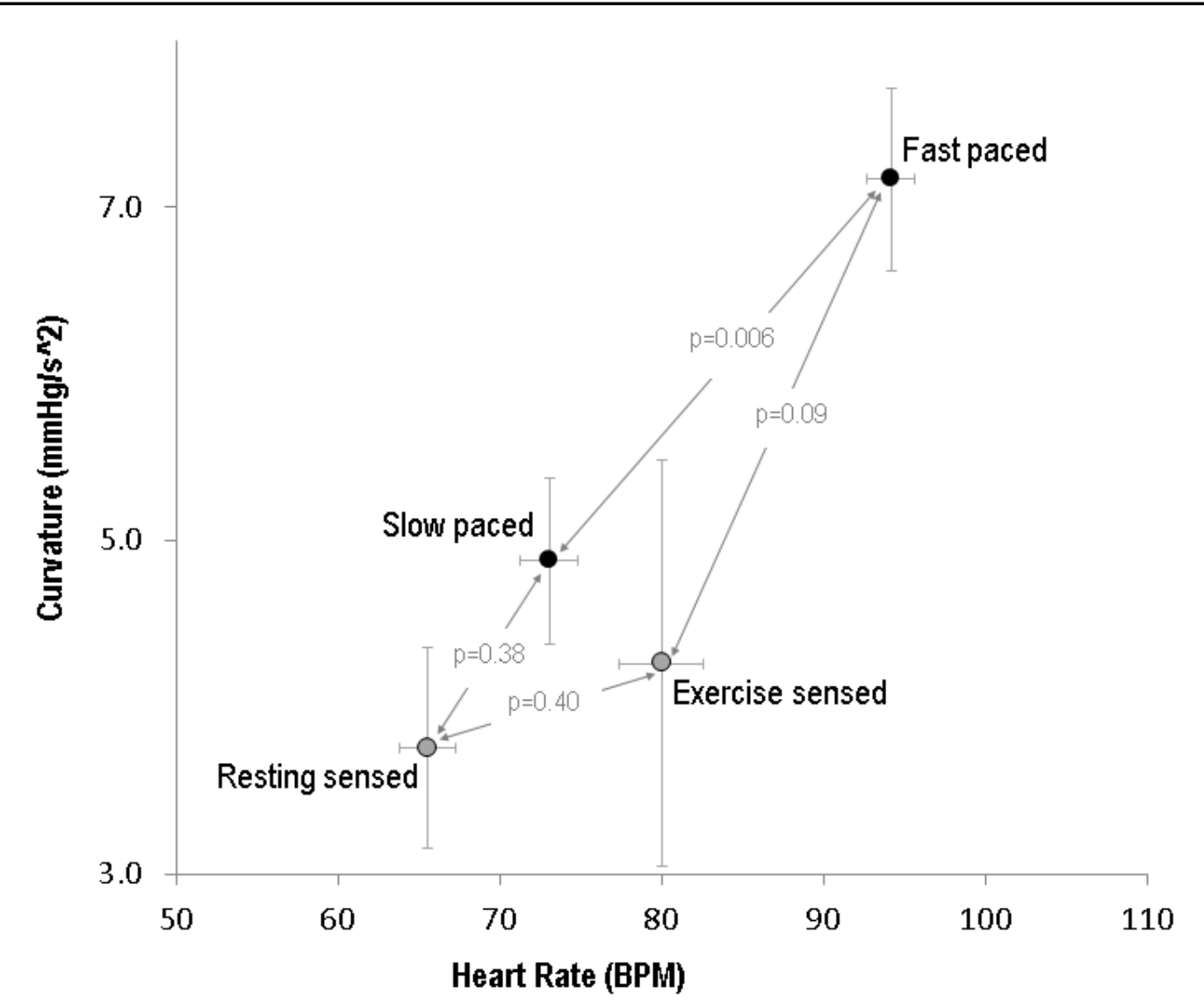

Figure 3: Contributions of atrial pacing mode and of increase in heart rate to the increase in curvature of the haemodynamic optimization parabola

The main contributor to the increase in curvature from resting to fast paced is the substantial increase in heart rate. 


\section{Discussion}

This study casts light on why it is easier to establish a precise (i.e. reproducible) AV optimum at fast paced heart rates than in the resting state. It appears that both components, imposition of atrial pacing and the substantial increase in heart rate, play a role in improving of the optimization process. However, the effect of atrial pacing is greater.

\section{Elements that could improve signal-to-noise ratio}

The improvement in signal-to-noise ratio is predominantly due to an increase in signal. However, in retrospect we realise that even the increase in signal has two distinct mechanisms. First, and more obvious, this can arise by the shape of the haemodynamic response changing from a shallow parabola to a steep parabola as we have previously reported ${ }^{4}$ to occur with increase in paced heart rate. Expressed briefly, as heart rate is raised it is diastole rather than systole whose duration is shortened. Time for filling is more exquisitely sensitive to reduction of cycle length. This effect is illustrated in Figure 4.

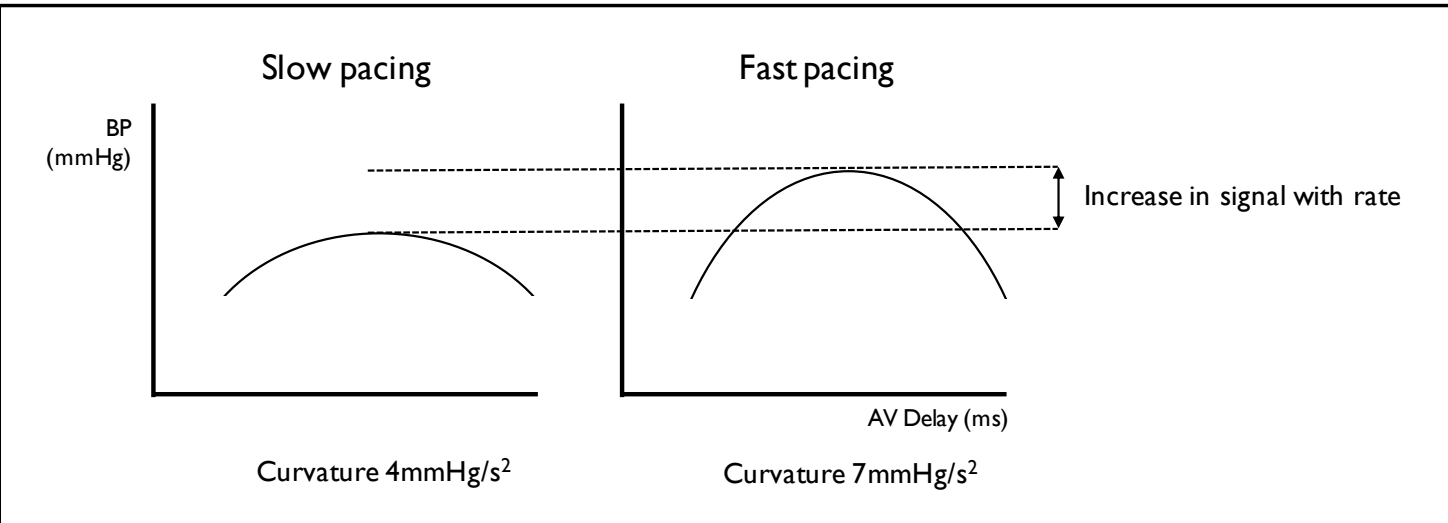

Figure 4: Increase in signal moving from slower to faster atrial pacing

Increase in signal is seen moving from slow atrial pacing to fast atrial pacing across a given range of AV delays. This is reflected in an increase in the curvature of the underlying parabolic relationship between AV delay and haemodynamic response.

There is a second, more subtle mechanism. Our protocol used the same range of programmed AV delays for sensed and paced. Thinking more deeply about the physiological consequence of using the same range for 
sensed and paced reveals that these would be addressing different ranges of the spectrum of mechanical atrioventricular delay.

The same programmed electrical AV delay, creates a shorter left mechanical AV delay during atrial paced than atrial sensed biventricular pacing (Figure 5). This "sensed-paced difference" at rest is in the region of $64 \mathrm{~ms}^{5}$ and can be attributed to three factors: atrial sensing delay, atrial pacing latency, and differences in intra/interatrial conduction between atrial sensing and pacing 5 .

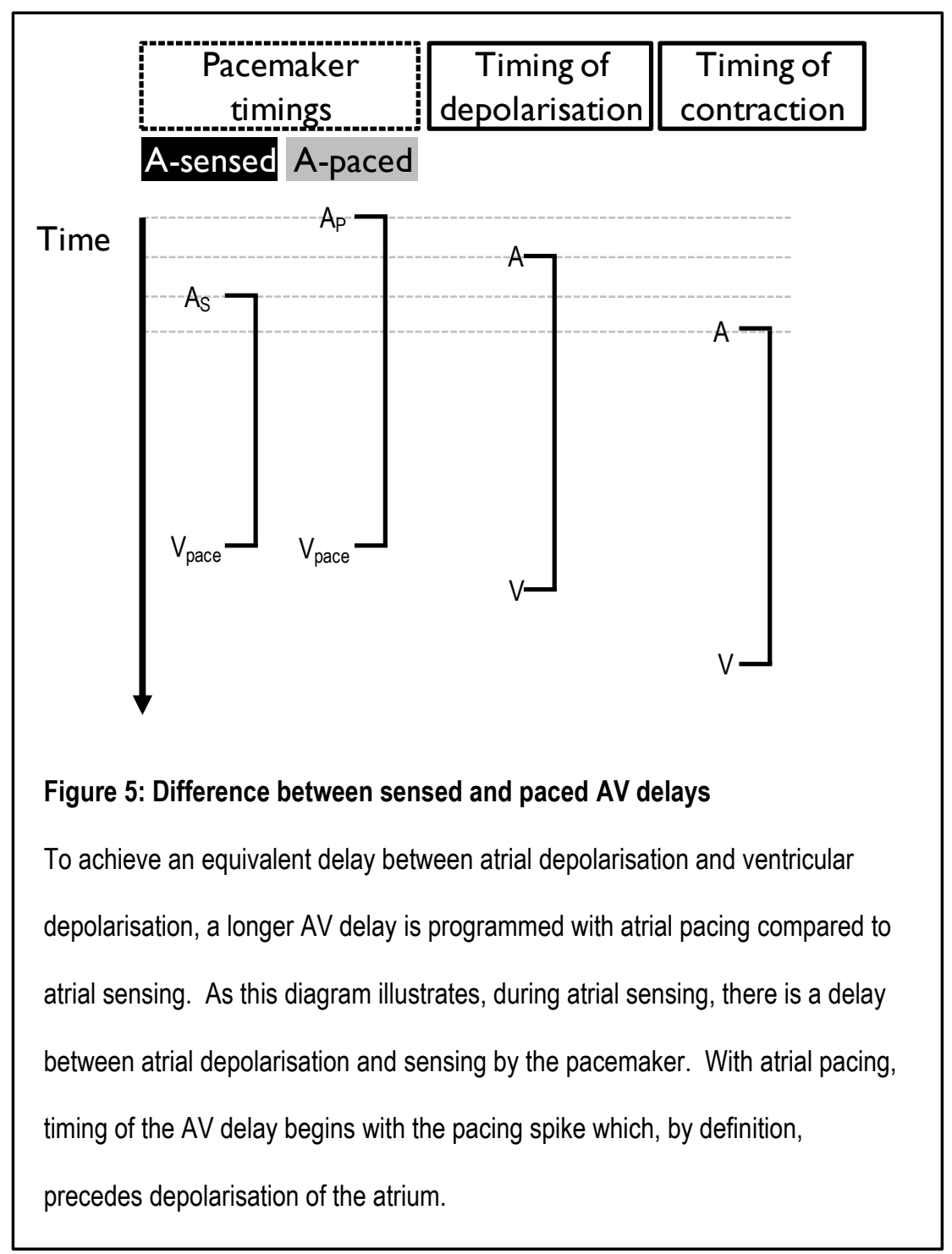

When the same range of programmed values is examined under atrial pacing as was examined under atrial sensing (Figure 6) the spectrum of mechanical delays being examined includes some shorter values that lead to 
poorer haemodynamics. Thus, when signal is defined as the maximum minus minimum SBP, the signal is larger. The curvature of the response curve, which for a parabola is consistent in all parts of the spectrum, is not detectably affected by this mechanism (Figures 3 and 6).

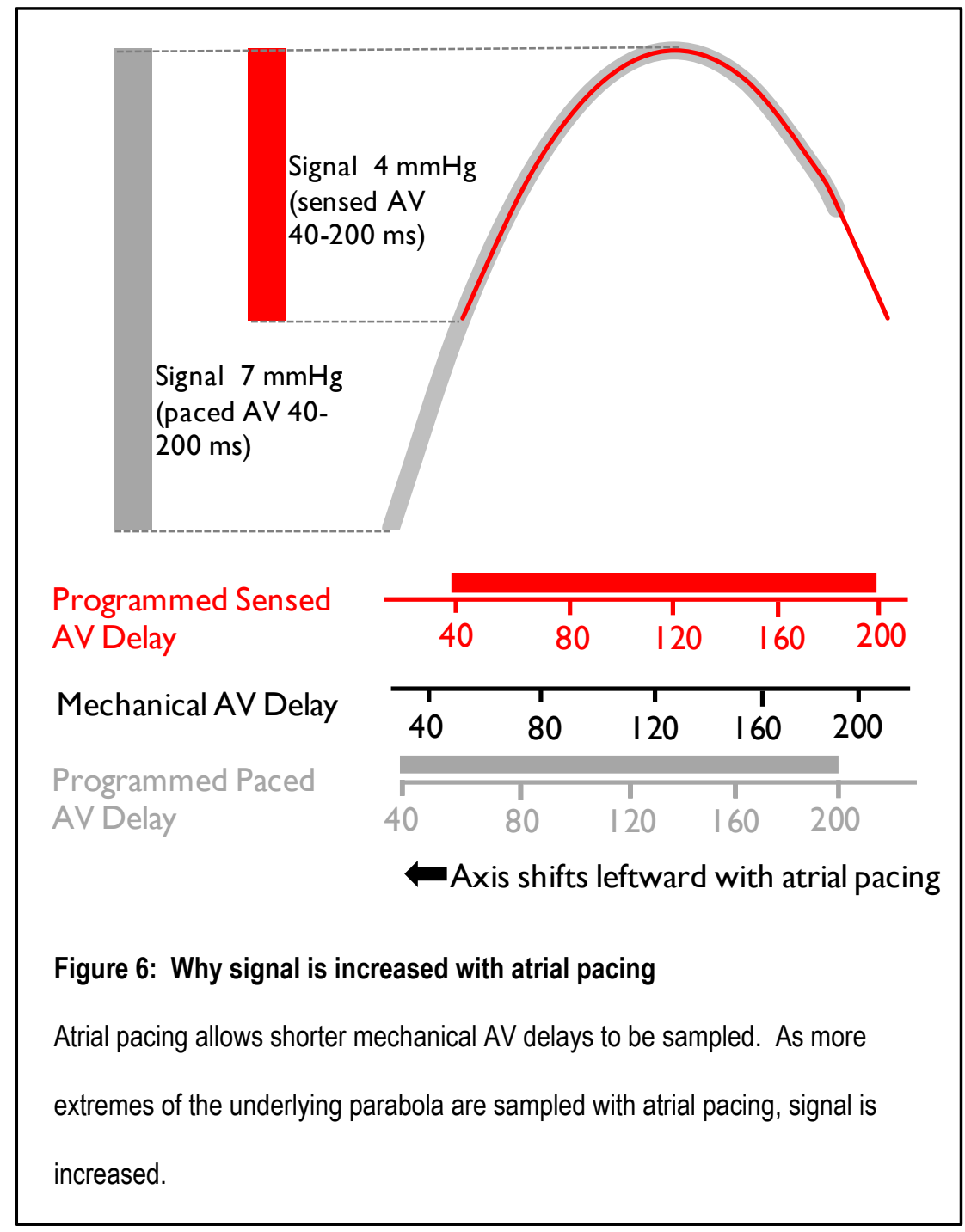

Our study suggests that increasing heart rate through exercise during atrial sensed optimizations does not improve the measured signal nor improve the ICC to a useful extent. Elevating heart rate by exercise achieved a lower increment in heart rate than by fast pacing. Moreover, the range of mechanical AV delays accessed by a given range of programmed AV delays is rightward (i.e. towards higher mechanical AV delays) for atrially sensed rather than atrially paced optimizations. These two effects together may explain why elevating heart rate by exercise did not increase signal to the extent seen with fast atrial pacing. 


\section{Clinical implications}

To optimize reliably, signal must be maximised and noise minimised. Increasing the number of replicate acquisitions decreases the impact of noise ${ }^{6}$. However, without automation this can be time consuming, and expensive. Additionally, until noise becomes smaller than the signal, information content may not begin to improve $^{6}$.

Our study suggests that increasing heart rate through exercise during atrial sensed optimizations does not improve the measured signal nor improve the ICC to a useful extent. Instead, atrial pacing is the most effective means of maximising information content.

We have previously reported ${ }^{4}$ that performing optimizations at faster atrially paced heart rates increases signal. This study indicates that atrial pacing itself is the predominant factor, contributing $78 \%$ of the improvement observed. This results from a greater range of tested mechanical AV delays in paced compared with sensed optimizations. Graphically on Figure 6 this is represented by reaching further left on the haemodynamic response curve. Adapting protocols to maximise signal to noise using an approach such as in this study has the potential to improve the clinical efficacy of optimization.

\section{Study limitations}

This study was only designed to investigate the effect of atrial pacing and heart rate on information content of haemodynamic optimization. It was not a study of the clinical benefits of optimization.

Blood pressure was measured non-invasively in the finger rather than invasively in the aorta although previous studies have shown good agreement between changes in non-invasive and invasive BPs in optimization protocols $^{8}$.

We did not study multiple heart rates but instead only a slow heart rate and a fast heart rate for each of atrial sensed and atrial paced. We do not know if the trends observed would continue to even higher or lower heart rates. 
We did not study VV delay in this particular study. For VV delay we would not expect there to be a change in the range of VV delays tested by moving from sensed to paced. In this setting only the effect of modifying heart rate could be studied.

Most of our patients were on beta blockers. This potentially prolongs a patient's PR interval. However, we would not expect this to affect our findings, as the difference in testable ranges in AV delay would remain consistent.

\section{Conclusions}

The act of atrial pacing itself, as opposed to an increase in heart rate, is the principle contributor to the large increase in ICC observed with haemodynamic optimisation at higher atrially paced rates. Optimising during atrial pacing enables the testing of shorter mechanical AV delays, which cannot be tested during atrially sensed heart rates; this gives a wider range of blood pressure changes that explains the improved information content. 


\section{Funding}

This work was supported by the British Heart Foundation to SMAS, PP, AK, ZIW, and DPF [grant numbers FS/11/92/29122, PG-08-114-25766, SP/10/002/28189, FS/08/027/24763, FS/13/44/30291, FS/10/038].

\section{Acknowledgements}

The authors are grateful for infrastructural support from the National Institute for Health Research (NIHR) Biomedical Research Centre based at Imperial College Healthcare NHS Trust and Imperial College London.

\section{Conflicts of Interest}

Darrel Francis is a consultant to Medtronic and Sorin. Zachary Whinnett acts as a consultant to St Jude Medical and Medtronic. 


\section{References}

1. Daubert J-C, Saxon L, Adamson PB, Auricchio A, Berger RD, Beshai JF, et al. 2012 EHRA/HRS expert consensus statement on cardiac resynchronization therapy in heart failure: implant and follow-up recommendations and management. Hear Rhythm 2012;9:1524-76.

2. Brignole M, Auricchio A, Baron-Esquivias G, Bordachar P, Boriani G, Breithardt O-A, et al. 2013 ESC Guidelines on cardiac pacing and cardiac resynchronization therapy: The Task Force on cardiac pacing and resynchronization therapy of the European Society of Cardiology (ESC). Developed in collaboration with the European Heart Rhythm Association (EHRA). Europace 2013;15:1070-118.

3. Whinnett ZI, Sohaib SMA, Mason M, Duncan E, Tanner M, Lefroy D, et al. Multicenter Randomized Controlled Crossover Trial Comparing Hemodynamic Optimization Against Echocardiographic Optimization of AV and VV Delay of Cardiac Resynchronization Therapy: The BRAVO Trial. JACC Cardiovasc Imaging 2018;

4. Whinnett ZI, Davies JER, Willson K, Chow AW, Foale RA, Davies DW, et al. Determination of optimal atrioventricular delay for cardiac resynchronization therapy using acute non-invasive blood pressure. Europace 2006;8:358-66.

5. Whinnett ZI, Briscoe C, Davies JER, Willson K, Manisty CH, Davies DW, et al. The atrioventricular delay of cardiac resynchronization can be optimized hemodynamically during exercise and predicted from resting measurements. Hear Rhythm 2008;5:378-86.

6. Pabari PA, Willson K, Stegemann B, Geldorp IE van, Kyriacou A, Moraldo M, et al. When is an optimization not an optimization? Evaluation of clinical implications of information content (signal-tonoise ratio) in optimization of cardiac resynchronization therapy, and how to measure and maximize it. Heart Fail Rev Springer US; 2011;16:277-90.

7. Whinnett ZI, Davies JER, Willson K, Manisty CH, Chow AW, Foale RA, et al. Haemodynamic effects of changes in atrioventricular and interventricular delay in cardiac resynchronisation therapy show a consistent pattern: analysis of shape, magnitude and relative importance of atrioventricular and interventricular delay. Heart 2006;92:1628-34.

8. Kyriacou A, Pabari PA, Whinnett ZI, Arri S, Willson K, Baruah R, et al. Fully automatable, reproducible, noninvasive simple plethysmographic optimization: proof of concept and potential for implantability. Pacing Clin Electrophysiol 2012;35:948-60.

9. Whinnett ZI, Nott G, Davies JER, Willson K, Manisty CH, Kanagaratnam P, et al. Maximizing 
2

4

5

6

7

efficiency of alternation algorithms for hemodynamic optimization of the AV delay of cardiac resynchronization therapy. Pacing Clin Electrophysiol 2011;34:217-25.

10. Whinnett ZI, Davies JER, Nott G, Willson K, Manisty CH, Peters NS, et al. Efficiency, reproducibility and agreement of five different hemodynamic measures for optimization of cardiac resynchronization therapy. Int J Cardiol 2008;129:216-26.

11. Davies LC, Francis D, Jurák P, Kára T, Piepoli M, Coats AJ. Reproducibility of methods for assessing baroreflex sensitivity in normal controls and in patients with chronic heart failure. Clin Sci (Lond) 1999;97:515-22.

12. Francis DP. Precision of a Parabolic Optimum Calculated from Noisy Biological Data, and Implications for Quantitative Optimization of Biventricular Pacemakers (Cardiac Resynchronization Therapy). Appl Math Scientific Research Publishing; 2011;02:1497-506.

13. Francis DP. How to reliably deliver narrow individual-patient error bars for optimization of pacemaker AV or VV delay using a \&quot;pick-the-highest\&quot; strategy with haemodynamic measurements. Int J Cardiol 2013;163:221-5. 